\title{
La gobernanza del riesgo en América Latina y la dimensión política de los desastres
}

\author{
Risk Governance in Latin America and the Political \\ Dimension of Disasters
}
A governança do risco na América Latina e a dimensão política dos desastres

\author{
Deysi Ofelmina Jerez-Ramírez \\ Universidad de Ciencias y Artes de Chiapas. Chiapas, México \\ deysiojerezramirez@gmail.com / deysi.jerez@unicach.mx \\ https://orcid.org/0000-0002-7033-7667
}

\section{Rogelio Josué Ramos-Torres}

Centro de Investigaciones y Estudios Superiores en Antropología Social. Ciudad de México, México r.ramos@ciesas.edu.mx

https://orcid.org/0000-0002-1080-4454

DOI: https://doi.org/10.32719/25506641.2022.11.9

Presentado: 13 de abril de 2021 - Revisado: 12 de mayo de 2021

Aceptado: 7 de junio de 2021 - Publicado: 1 de enero de 2022

Artículo de investigación
Licencia Creative Commons 


\section{Deysi Ofelmina Jerez-Ramírez y Rogelio Josué Ramos-Torres}

\section{Resumen}

La gobernanza para la reducción del riesgo de desastres (RRD) es un enfoque que, acuñado en el ámbito de la cooperación internacional, se ha ido extendiendo e incorporando a las diferentes agendas nacionales de los Estados miembros. Sin embargo, su génesis y difusión reflejan tensiones y vacíos desde los cuales se puede cuestionar la real efectividad de este modelo como estrategia preventiva. El presente artículo revisa los orígenes de la gobernanza del riesgo, observando las distintas acepciones a que puede ser sujeto y problematizando el concepto frente a las condiciones de vulnerabilidad que plantea el contexto latinoamericano. A partir de la mirada de la ecología política se señala que, dentro de este contexto, las relaciones de poder locales son uno de los puntos ciegos de dicha propuesta, lo cual se encuentra estrechamente vinculado con la ausencia de una concepción amplia de la política y lo político, dentro de los elementos que configuran la agenda de la gobernanza para la RRD. El método utilizado corresponde a la investigación documental.

Palabras clave: gobernanza, reducción del riesgo de desastres, relaciones de poder, vulnerabilidad, política.

JEL: Q54 Clima, desastres naturales y su gestión, calentamiento global.

\section{Abstract}

Governance in disaster risk reduction (DRR) is a strategy that has been coined in the field of international cooperation, thereby expanding into the different national agendas of the member states. However, its genesis and dissemination reflect tensions and gaps that question the real effectiveness of this model as a preventive strategy. This article analyzes the origins of risk governance, observing the different meanings attributed to it and problematizing the concept given the conditions of vulnerability posed by the Latin American context. From the perspective of political ecology, we have identified that, within this context, local power relations are one of the blind spots of said proposal, which is closely linked to a lack of general understanding of politics and political matters within the elements that make up the DRR governance agenda. The method used for this study is a documentary research approach.

Keywords: governance, disaster risk reduction, power relations, vulnerability, politics.

JEL: Q54 Climate, natural disasters and their management, global warming.

\section{Resumo}

A governança para a redução do risco de desastres (RRD) é um enfoque que, ideado no âmbito da cooperação internacional, tem ido estendendo-se e incorporando-se às diferentes agendas nacionais dos estados membros. Contudo, sua gênese e difusão refletem tensões e vazios desde os quais pode-se questionar a real efetividade deste modelo como estratégia preventiva. $\mathrm{O}$ presente artigo revisa as origens da governança do risco, observando as distintas acepções a que pode ser sujeito e problematizando o conceito frente às 
condições de vulnerabilidade que planteia o contexto latino-americano. A partir da visão da ecologia política sinaliza-se que, dentro deste contexto, as relações de poder locais são um dos pontos cegos de dita proposta, o qual se encontra estreitamente vinculado à ausência de uma concepção ampla da política e o político, dentro dos elementos que configuram a agenda da governança para a RRD. O método utilizado corresponde à investigação documental.

Palavras-chave: governança, redução de risco de desastres, relações de poder, vulnerabilidade, política.

JEL: Q54 Clima, desastres naturais e sua gestão, aquecimento global.

\section{Introducción}

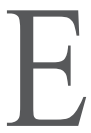

n el marco de la conmemoración del Día Internacional para la Reducción del Riesgo de Desastres de 2020, los debates ocurridos a nivel global en torno a la agenda de reducción de riesgo de desastres (RRD), se centraron en las estrategias de la buena gobernanza y los actuales desafíos para la implementación de la prioridad de la acción 2 de la plataforma del Marco de Acción de Sendai.

No obstante, el Informe de Evaluación Regional sobre el Riesgo de Desastres en América Latina y el Caribe, presentado por la Oficina de las Naciones Unidas para la Reducción del Riesgo de Desastres (ONU 2021), reflexiona sobre la importancia de replantear dicho enfoque a fin de superar, finalmente, el tratamiento reactivo en la RRD e intervenir en las causas de fondo derivadas del actual modelo de desarrollo.

Las intenciones, desde la agenda internacional, de incidir mediante el esquema de gobernanza del riesgo en los procesos de decisión a nivel regional y local, se fundamentan en una simplicidad que no corresponde con la compleja dinámica territorial de los contextos latinoamericanos. Los conceptos colonizantes (Revet 2011; Macías 2015; Frenkel 2019) de los organismos multilaterales para la RRD siguen impulsando la intervención externa en la región, minimizando el papel de los actores locales. Simultáneamente, las agendas de cooperación internacional sobre el tema operan desde el desconocimiento de las relaciones de poder y los procesos históricos de resistencia como factores estructurales para la materialización del riesgo y la construcción social de la prevención, respectivamente. 
La gobernanza es una estrategia que conjuga una serie de acciones a diferente nivel (técnico, social, ambiental, jurídico, seguridad, económico, político). Para el presente estudio, interesa indagar en las particularidades sociales y políticas de este enfoque, por lo que se busca dimensionar, mediante el modelo de la ecología política y el paradigma de vulnerabilidad, la real incidencia del enfoque de gobernanza en el contexto latinoamericano, develando la paradoja que subyace al tratamiento apolítico de los desastres. El análisis se centra, además, en los retos no revisados de la gobernanza para la RRD, a partir del rastreo de ciertas tensiones que particularizan la escena regional, a saber: a) relaciones de dominación-resistencia; b) pluralidad de actores contendientes por el poder; y c) condiciones de riesgo y vulnerabilidad generadas desde el sistema político-económico.

\section{Método}

Se trata de un estudio descriptivo-interpretativo que trabaja con las particularidades que se develan en el enfoque de gobernanza del riesgo y el concepto de desastres, al analizarse en el contexto latinoamericano de vulnerabilidad. El método utilizado para el desarrollo del artículo es la investigación documental (Rojas 1995). Se realizó una revisión selectiva a fuentes documentales, cuyos datos fueron sistematizados y organizados, mediante fichas de trabajo, en categorías que responden a tres niveles de información: enfoques teórico-conceptuales, enfoques institucionales y temas asociados.

\section{Antecedentes de la gobernanza del riesgo}

Durante la década de 1990, la declaración de las Naciones Unidas (ONU 1989) del Decenio Internacional para la Reducción de los Desastres — mal llamados- Naturales (DIRDN) impulsó una serie de principios rectores en el tema de la prevención y la atención. La incorporación de dichas directrices presentó como principal desafío la reestructuración de los esquemas y procesos para la toma de decisiones sobre mitigación de efectos y vulnera- 
bilidades, asuntos en los que, de aquí en adelante, el papel de la comunidad tendría mayor relevancia. Llama la atención cómo incluso en el contexto de globalización y apertura de las fronteras mundiales, la respuesta local y territorialmente específica reclamaba un lugar en la agenda de cooperación internacional para sumarse a los retos del decenio, aunque esa incorporación, cabe señalar, no ha sido del todo efectiva.

En 2005, la Conferencia Mundial sobre la Reducción de Desastres permitió la creación del Marco de Acción de Hyogo (MAH) como un plan a largo plazo en materia de RRD. En el MAH (2005-2015) la participación comunitaria mantuvo vigencia, pero ahora como parte de un concepto más ambicioso: la gobernanza del riesgo.

De acuerdo con el Informe de Desarrollo Humano de 2019, la gobernanza $^{1}$ - gobernanza o governance, que proviene del francés y se tradujo al inglés (Sánchez 2006) — no es otra cosa que "la forma en que los distintos agentes que conforman la sociedad negocian para llegar a acuerdos" (PNUD $2019,13)$, crear políticas y establecer normas. En la materia que nos interesa, el documento de la OCDE (2014) define a la gobernanza positiva de los riesgos como una inversión que es clave y estratégica para que los países no comprometan su crecimiento financiero a largo plazo como consecuencia de fenómenos naturales, fallas tecnológicas o pandemias.

El Marco de Acción de Sendai, actual plataforma internacional que sustituyó al MAH, presenta la gobernanza para la reducción de los desastres como una acción prioritaria:

La gobernanza del riesgo de desastres en los planos nacional, regional y mundial es de gran importancia para una gestión eficaz y eficiente del riesgo de desastres a todos los niveles. Es necesario contar con claros objetivos, planes, competencia, directrices y coordinación en los sectores y entre ellos, así como con la participación de los actores pertinentes. Por lo tanto, el fortalecimiento de la gobernanza del riesgo de desastres para la prevención, mitigación, preparación, respuesta, recuperación y rehabilitación es necesario y fomenta la colaboración y las alianzas entre mecanismos e instituciones en la aplicación de los instrumentos pertinentes para la reducción del riesgo de desastres y el desarrollo sostenible. (ONU 2015, 17)

1. Se deriva de las ciencias políticas y de la administración pública en el marco del debate sobre el ser y el deber ser del Estado, a inicios de la década de 1980. 
La evolución del estudio de los desastres, así como los cambios representativos en el discurso académico del riesgo, anteceden a la incorporación de nuevos conceptos que, como la gobernanza, hacen referencia a la dimensión social de la problemática, más allá de las dinámicas físico-naturales. El cambio de paradigma impulsado en las investigaciones sobre el tema en la primera mitad del siglo XX, pero puntualmente para Latinoamérica a finales de 1980, permitió abandonar paulatinamente la visión fisicalista ${ }^{2}$ de los desastres dentro del análisis académico, una cuestión pendiente en el discurso institucional. El enfoque social, como se conoce a este salto analítico-explicativo, se alimentó de diversas teorías y metodologías provenientes de las ciencias sociales y de enfoques que marcarían diferentes etapas del quehacer científico sobre la gestión del riesgo.

Los estudios implementados en el ámbito de la intervención comunitaria abrieron paso a una serie de debates que cuestionaron el monopolio tecnocrático en la toma de decisiones para la gestión del riesgo. La academia, desde las ciencias sociales, se propuso aportar planteamientos metodológicos para la participación social crítica en el direccionamiento de políticas públicas, superando la participación representativa y los roles consultivos designados tradicionalmente a la ciudadanía.

Adicionalmente, el aporte de los teóricos del riesgo del siglo XX marcó otro antecedente fundamental en el camino hacia el enfoque de la gobernanza. Las obras de Beck (2006) y Luhmann (2006) se ocuparon de las dimensiones y elementos de un riesgo sistémico configurado como la esencia de la modernidad. Los efectos involuntarios de la acción humana se evaluaron como una acumulación histórica de vulnerabilidades imposibles de abordar desde una "solución biográfica" (Beck 2006, 137).

En este contexto, las causas antropogénicas cobraron importancia para la interpretación heurística del problema. El Plan de Acción de Yokohama (ONU 1994) se enfocó principalmente en los impactos de amenaza natural, por lo que la incorporación del riesgo asociado al desarrollo tecnológico y a la interacción sociedad-entorno fue una deuda a saldar en los documentos de Hyogo y Sendai.

2. Es el paradigma que se rige por los conocimientos y metodologías de las ciencias exactas para la interpretación de las causas de los desastres, frecuentemente relacionadas con un origen natural y físico. 
Rápidamente se entendió que la capacidad de coordinar acciones y recursos para la RRD, en función del bien común, parte del reconocimiento de las fallas y contradicciones del propio sistema. El cambio climático, las pandemias y los desastres nucleares son un claro ejemplo de las consecuencias del mal gobierno que ha caracterizado a las sociedades industriales. No obstante, la falta de una revisión profunda de las causas estructurales de los desastres por parte de los actores, organizaciones e instituciones que conforman el sistema internacional para la RRD, sigue priorizando los intereses de reducidos sectores políticos y sociales que son ajenos a la dinámica local. Aquí se centra una de las principales críticas al enfoque de la gobernanza y de otros esquemas definidos como colonizantes.

\section{Problematización del enfoque de gobernanza: las agendas colonizantes de la reducción del riesgo de desastres}

La sobreproducción de conceptos y la continua actualización de agendas sobre RRD, se han popularizado desde finales del siglo pasado en el escenario de la acción internacional. Este hecho es defendido al reconocer al riesgo y a los desastres como problemáticas multicausales que, además, se complejizan al incorporarse en los objetivos globales del desarrollo -Declaración del Milenio y Agenda 2030 - El concepto de seguridad humana acuñado por el PNUD en 1994 (PNUD 1994) que configuró medidas más humanitarias que aquellas impulsadas bajo el concepto de Homeland Security de los Estados Unidos o la Estrategia Europea de Seguridad (Ortega y Font 2012), centró interés en las condiciones mundiales de pobreza como una amenaza global que requería de la intervención de la cooperación internacional. El impacto generalizado de los desastres en gran parte de las economías en desarrollo también justificó mantener el tema de la reducción del riesgo en la esfera de los organismos multilaterales.

A partir del enfoque de gobernanza se avaló el diagnóstico respecto a los débiles instrumentos estatales para hacer frente a estas problemáticas transnacionales y se reiteró la necesidad de la ayuda externa. 
Diversos trabajos que corresponden a una etapa más reciente de producción académica en torno a la RRD, realizaron una crítica a los efectos colonizantes de ciertos conceptos (Revet 2011; Macías 2015; Frenkel 2019) en las dinámicas locales de afrontamiento del riesgo y apoyaron el debate académico sobre la institucionalización fuera de lo nacional.

Revet (2011, 539), por ejemplo, centró el análisis en las formas de legitimización de lo que denomina proceso de internacionalización de los desastres; es decir, la "dinámica que transforma situaciones sociales ancladas en ámbitos locales o nacionales en situaciones de las que se hacen ahora cargo los actores que superan las fronteras nacionales y las referencias estatales". En este espacio de apropiación internacional, las formas de legitimización descansan en argumentos como la incapacidad de los Estados ante las crisis globales o la especialización de organismos multilaterales en el abordaje de temas públicos (Revet 2011).

La promesa de la gobernanza en las últimas décadas apuntó hacia el fortalecimiento de las capacidades nacionales a partir de la reestructuración de los procesos de decisión, lo cual se vislumbró como una oportunidad para la participación social efectiva. Empero, el paradigma de la internacionalización cambia a los actores sociales, económicos y políticos locales por agencias y agentes externos; además, el argumento de la especialización excluye los conocimientos no técnicos de las comunidades, reclamando la certeza científica en torno a los objetivos de RRD. La participación comunitaria sigue careciendo de la institucionalización de la que ya hace tiempo presume la intervención extranjera.

La crítica al enfoque de gobernanza se presenta en el trabajo de Pereira y Raju (2020), quienes señalan la importancia de contextualizar aquellos esquemas provenientes de la agenda internacional para evaluar su real efectividad. Los autores se basan en la perspectiva del neoextractivismo (Azamar y Ponce 2015; Svampa 2019) para explicar las implicaciones del patrón político-económico de explotación intensiva de recursos naturales en el desarrollo de desastres y su posible prevención. Acuden, igualmente, al paradigma de vulnerabilidad para caracterizar el patrón político-ecológico que funciona en Latinoamérica y que sigue aumentando la exposición de la región a amenazas diversas. El concepto de neoextractivismo ha resultado de gran utilidad en la nueva corriente crítica sobre RRD; empero, dichos trabajos — específi- 
camente en el tema que aquí interesa - han tardado en reconocer a la región como un territorio histórico de resistencia que ha hecho frente a las dinámicas desiguales impulsadas desde el sistema hegemónico, dinámicas que median en la interacción sociedad-entorno. La incorporación de la relación dominación-resistencia, por ende, resulta crucial en el análisis regional sobre gobernanza del riesgo.

Por su parte, la investigación de Frenkel (2019), incluida también en esta reciente etapa de producción crítica, advierte sobre otro asunto preocupante. La incorporación de los desastres en los diferentes esquemas de securitización impulsados desde los principales organismos multilaterales, ha sido la puerta de entrada para diferentes conceptos y enfoques que propenden por la anulación de la heterogeneidad de actores y perspectivas. De igual forma, el manejo de los desastres como asunto de seguridad parece relacionarse con el resurgimiento de otras corrientes que parecían superadas: el paradigma fisicalista retorna al invocar nuevamente el argumento de la ciencia y la expertise, así entonces, la práctica tecnocrática es pensada como un literal "gobierno de los técnicos" (Jerez-Ramírez 2016, 3).

Un nuevo asalto se dio en la siguiente Conferencia de ministros de Defensa de las Américas (2012), cuando Chile presentó una nueva propuesta de mecanismo hemisférico bajo el nombre de Sistema de Cooperación de Asistencia Humanitaria (SICAHUM). Más allá de que contenía algunas diferencias respecto a las iniciativas anteriores, la nueva propuesta se mantenía dentro del esquema fisicalista de atención a desastres, al desestimar el rol de las comunidades locales y privilegiar las acciones multilaterales e internacionales a posteriori de los acontecimientos. (Frenkel 2019, 193-194)

La priorización de las acciones de atención de la emergencia, antes que la prevención del desastre es un ámbito en el que la idea de gobernanza del riesgo no puede operar, por lo que resultaría conveniente revisar un enfoque que, además, parece consolidarse como una paradoja. La gobernanza es a todas luces un concepto político que nace de la evaluación de las capacidades del Estado para dirigir las decisiones y acciones frente a una problemática - los desastres - que, no obstante, se siguen manejando como un asunto apolítico. Este tema se profundizará a continuación. 


\section{Las relaciones de poder local: ¿dimensión invisibilizada por las estrategias de gobernanza para la RRD?}

La gobernanza para la RRD y su adopción, en virtud de tratados y agendas internacionales por países a lo largo y ancho del mundo, se ha prestado también a interpretaciones que han tenido como efecto el desvanecimiento conceptual del término. En la práctica, esto repercute en una falta de claridad que impide el conocimiento de las formas en que los gobiernos y las sociedades negocian los acuerdos para la participación y la toma de decisiones, lo que redunda en una ausencia de medidas concretas para la reducción de los riesgos (Sandoval y Sarmiento 2018).

La inserción de la gobernanza como vía para la RRD se encuentra, según sus propias consideraciones (ONU 2015), en concordancia con el paradigma de la vulnerabilidad, según el cual esta se determina por la forma en que la sociedad interactúa a través de procesos cruzados por 1o económico y lo político (Blaikie et al. 1996). Es en estos intercambios en donde las sociedades distribuyen y ordenan aspectos tales como la planificación urbana o territorial, el trabajo, el nivel de ingresos, el sistema de producción, el acceso a la salud, el acceso a la educación y a la información, entre otros.

Por lo tanto, es difícil el éxito de una estrategia preventiva y la creación de medidas concretas, incluso en planos de coordinación, si no se cuenta con un grado de conocimiento suficiente a nivel territorial que permita entender el funcionamiento de estas interacciones, reconociendo que los territorios son espacios atravesados por relaciones de poder (Raffestin y González 2013), y que todo esfuerzo organizativo, por exitoso que sea, nunca dejará de ser desafiante (Wolf 1990).

La ecología política (Blaikie 1985; Bryant y Bailey 1997) y más puntualmente la ecología política de los desastres (Bravo 2018; Pelling y Dill 2009; Romero y Romero 2015), han puesto especial énfasis en este aspecto. Dichos enfoques reconocen el imperio que tienen las lógicas mercantiles en la modernidad, en donde se ha sometido a la naturaleza a un proceso de instrumentalización para reducirla a simples espacios de explotación, gene- 
rando una serie de conflictos a diferente escala. En ese sentido, la relación con los entornos y sobre todo el acceso diferenciado a los recursos naturales como producto de las relaciones de poder, es el origen de tensiones permanentes, entre una gama de actores, en la que el Estado es solamente uno más de ellos. Estas relaciones son clave para el análisis de las causas sociales de fondo detrás de los desastres, pues es ahí en donde se determina el grado de vulnerabilidad de grupos y personas.

En ese sentido, no es suficiente mapear a las fuerzas estructurales que, en su desenvolvimiento, menoscaban capacidades de personas y comunidades, tales como los sistemas de gobierno (Cohen y Werker 2008) o los neoextractivismos (Pereira y Raju 2020). Para los esfuerzos de RRD en general, igual de importante es bajar el lente de observación a lo territorial y observar ahí las dinámicas de fuerza que pueden abrevar o no de estructuras de poder más grandes, pero que tienen una incidencia en los intercambios productores de riesgos y vulnerabilidades.

En este orden de ideas, la dimensión política cobra una importancia determinante, pues constituye "la vía para transitar de un mundo global regido por el poder unificador del mercado hacia la construcción de un mundo diverso orientado por una ontología de la diversidad, de la diferencia y la otredad" (Leff 2017, 138). Bajo esta premisa, los efectos de una efectiva campaña de prevención de RRD deben tener también un impacto en esas relaciones de poder, modificando o inhibiendo los aspectos que generan vulnerabilidades sociales.

Por estas razones, resulta cuestionable que los procesos para el buen gobierno de la RRD se organicen a partir de una lógica externa a los actores locales. La experiencia eficaz y práctica de la gobernanza debe construirse desde una mirada territorial concreta que devuelva el asunto de la reducción de riesgos a las agendas autónomas de las comunidades.

En el tratamiento de las vulnerabilidades, miradas más focalizadas y complejas del poder, permiten entender esa contraparte conceptual orgánica de la vulnerabilidad al que autores como Aguirre (2004) definen como poder de resistencia, y que otorga otro matiz histórico a esta dimensión y sus efectos dentro del entramado social, especialmente en América Latina, en donde a la par de los procesos coloniales de dominación, han caminado en el tiempo 
aquellos pasos de resistencia social que los pueblos han opuesto en aras de su libre determinación.

\section{Buscando la dimensión política en la gobernanza del riesgo desde la experiencia latinoamericana}

Como se ha señalado, la definición de gobernanza acuñada por el PNUD (2021) en el ámbito de la RRD, constituye el eje de una estrategia preventiva de atención basada en la integración y organización de una multiplicidad de actores. Esto coloca al tema dentro de la esfera de la política.

Sin embargo, la heterogeneización y el abuso que ha sufrido el término de gobernanza del riesgo a lo largo de los últimos años, dificulta la tarea de localizar sus propios alcances políticos. Estos claroscuros, lejos de favorecer su asentamiento, hacen posible la persistencia de las visiones fisicalistas que resultan tan favorables para que gobiernos y administraciones endosen a la naturaleza las responsabilidades que a ellos corresponden (Cardona 2001).

En suma, no queda claro la profundidad con la que el enfoque de la gobernanza para la RRD está viendo a la política y sus potencialidades, condicionando así su éxito en continentes como el latinoamericano, en donde la generación de desigualdades, propia del modelo de desarrollo capitalista, se encuentra estrechamente asociada con los desastres (Pelling y Dill 2009). En estas realidades, la vulnerabilidad no abandona su vigencia paradigmática para comprender y poder disminuir el riesgo, teniendo sobre todo en cuenta la colonialidad persistente (Toro 2017) en la región, visible a través de procesos como la explotación privada de los recursos naturales y la subalternidad de amplios sectores de la población que han sido históricamente determinantes para la configuración del orden sociopolítico (Alimonda 2015).

Significa que en los territorios latinoamericanos los promotores de la gobernanza tienen que hacer frente a una serie de tensiones particulares que retan la viabilidad de la estrategia. Una de estas tensiones está dada en el hecho de que las sociedades se encuentran estratificadas en gran medida bajo relaciones de dominación-resistencia, lo cual obstaculiza el espíritu colaborativo que la gobernanza promueve. A esto hay que agregar como elemento 
de tensión la presencia de actores contendientes por el poder que pertenecen a ámbitos diversos: legal (ONG) e ilegal (crimen organizado), internacional (transnacionales) y local (sindicatos, gremios), económico (empresas) y social (asociaciones civiles), entre otros.

El enfoque enfrenta aquí lógicas de desarrollo en las que la generación de riesgos y vulnerabilidades es parte estructural de los actuales sistemas políticos (Pelling y Dill 2009) y de producción (Delgado 2013), articulados ambos a un pensamiento hegemónico global (Toro 2017). Se hace referencia a entornos en los que la manipulación del riesgo como elemento sistémico de la vida cotidiana (Beck 2006), alimenta las dinámicas políticas y de mercado que sostienen a las hegemonías. Esto explica la preeminencia de las visiones fisicalistas en políticas públicas y estrategias institucionalizadas para la respuesta-atención a desastres en los países de la región (Rodríguez 2017), que proveen terrenos propicios para los asistencialismos y la construcción de sistemas de clientelas que extinguen lo político. De esta forma, las hegemonías aseguran la permanencia de un contrato social cuya esencia, pudiendo haber sido blanco de objeciones y controversia, sobre todo durante coyunturas críticas (Peeling y Dill 2009), conserva las mismas jerarquías y se mantiene en el mismo estado en que se encontraba antes de la crisis.

\section{La gobernanza del riesgo y los espacios de confrontación del poder}

La puesta en práctica de una gobernanza realmente asequible dentro del ámbito de la RRD requiere de una concepción de la política que deje de lado las pretensiones de consenso y recupere el antagonismo como momento fundacional. Pensar dentro del contexto latinoamericano - caracterizado por brechas de clase y relaciones asimétricas tan profundas - en la posibilidad de entendimientos o pactos armónicos, incluso en aras de procurar la salvaguarda de las poblaciones frente a los desastres, es caer en el mantenimiento del statu quo.

Lograr una lectura incluyente de la política, desde la cual se refleje realmente el interés de todos los grupos sociales, no es fácil. Los espacios en 
donde estos intereses son expresados varían de un grupo a otro y a menudo se sobreponen. Aunque el marco de acción vigente que es el Marco de Sendai 2015-2030 sugiere a los gobiernos atender la incorporación de grupos vulnerables y demás actores en la toma de decisiones para la posterior planificación de políticas, se ha documentado que en la práctica los ejercicios de atención al riesgo de desastres no solamente ignoran las condiciones específicas de los distintos colectivos, sino que no permiten la participación de ninguno de estos dentro de los procesos de decisión (Sosa y Vázquez 2014).

En estas realidades, puede ser un error esperar que las dinámicas de la política se revelen por sí mismas, pues los mecanismos del poder en contextos de dominación son por naturaleza despolitizantes y/o inhibidores de las expresiones subalternas. Es por ello necesario encontrar -o generar- dentro del universo local de relaciones, aquellos vértices y coyunturas en donde el poder es esgrimido y negociado.

Chantal Mouffe (1999) diferencia entre la política - momento de establecimiento de un orden que organiza la coexistencia humana-y lo político - asociado a una dimensión de antagonismo u hostilidad presente de manera inevitable en las relaciones humanas-. En esta idea, lo político es componente e inherente a la política, por lo que concebir a esta última únicamente como la suma de voluntades para conformar un orden social determinado, implica considerar la polis desestimando el polemos (Mouffe 1999) o pensar en la democracia desechando de antemano los antagonismos o desacuerdos que constituyen sus rasgos característicos.

Para esta autora, la figura del adversario ayuda a romper la falsa ilusión del espacio político como un terreno neutral - tal como se plantea desde las agendas de cooperación internacional - y pensarlo como un espacio cuya formación es expresión de relaciones de poder en permanente tensión. En ese sentido, un orden que se ostenta como democrático - requisito fundamental para la gobernanza - no neutraliza el poder, sino que aumenta los espacios en los que estas relaciones están abiertas a ser cuestionadas (Mouffe 1999) y confrontadas.

A este respecto, Ranciere, Panagia y Bowlby $(2001,9)$ dicen que ahí donde la expresión de un poder determinado coopte el espacio lanzando como mensaje: "sigue caminando, aquí no hay nada que ver", la política consiste en la posibilidad de transformar ese espacio en donde "no hay nada 
que ver", y reconfigurarlo de tal modo que puedan aparecer los individuos o grupos silenciados por relaciones de poder dispares.

En términos prácticos, esto significa que el enfoque de gobernanza del riesgo tendría que, idealmente, contar no solo con la capacidad de construir espacios de convergencia, sino que los diálogos ahí entablados se den en planos de equilibrio que permitan la expresión de todos los imaginarios sociales, evitando la primacía de aquellos que se manifiestan como hegemónicos. Uno de los objetivos de esos diálogos debe ser el de romper la idea de que la RRD es asunto exclusivo de unos pocos, técnicamente capacitados, e insertar la visión de todos aquellos a los que esos riesgos atañen. El desafío aquí no se encuentra tanto en prestar atención a las voces de todos los sectores, como en el diseño de una estrategia que asegure que esas voces se expresen libremente. Una puerta de entrada a esta posibilidad es la adopción de perspectivas teórico-metodológicas tales como la investigación-acción-participativa propuesta por Borda (1992) o la acción comunicativa de Habermas (2001). Puntualizando que, en contextos conflictivos como los ya descritos, este tipo de aproximaciones no son medidas acabadas o definitivas, sino un primer paso en el camino hacia el fortalecimiento de capacidades comunitarias frente a intereses externos.

\section{Conclusiones}

El actual marco internacional para la reducción del riesgo y los desastres, pese a la diversidad de enfoques, estrategias y acciones desplegadas desde los organismos multilaterales, se ha gestado en un escenario tan complejo como contradictorio, en donde el debate no ha llegado a la profundidad propuesta mediante el esquema de las ciencias sociales y se sigue manejando la problemática de los desastres como un asunto apolítico. Esta puede ser la razón más notable en el fallido intento por superar el paradigma fisicalista en la práctica latinoamericana.

El mosaico de enfoques para la RRD que se ha presentado en las últimas décadas en el ámbito de la cooperación internacional, finalmente se ha decantado por la gobernanza del riesgo, modelo que recientemente ha sido objeto de un amplio debate. 
La evolución/adopción del enfoque de gobernanza por parte de gobiernos y agencias en el mundo, obliga a revisar la categoría y a cuestionar su aplicabilidad en contextos determinados (Pereira y Raju 2020). Se destaca la interrogante sobre la forma en que las tensiones que acompañan al enfoque desde su surgimiento, más aquellas que las circunstancias particulares plantean, dificultan su efectiva puesta en marcha. Entre esas tensiones se encuentra la de ser un instrumento emanado de agendas de naturaleza políticamente neutral que, sin embargo, han adoptado un concepto que no puede negar dicha naturaleza, al estar directamente relacionado con las capacidades del Estado y los procesos de decisión que impactan directamente en los contextos locales.

Establecida la dimensión política de la gobernanza se complejiza el escenario, ya que la tensión, lejos de estar resuelta, se convierte en una paradoja: ¿puede un asunto apolítico (los desastres) ser materia de una estrategia política? Bajo este tenor, la gobernanza del riesgo se define como un modelo que nace del manejo neutral de los organismos internacionales para atender la problemática no política de la materialización del riesgo. Esta característica representa, en esencia, un grave obstáculo para su propio éxito.

En América Latina, el acceso desigual a los recursos naturales y las relaciones de poder ampliamente asimétricas operan como causas históricas de los procesos de desastre. Una mejor —o más incluyente - lectura de las panorámicas locales y sus correlativas relaciones de poder, podría ser llevada a cabo si se cuenta con una concepción de la política que supere su ubicación en espacios tradicionales, para incluir aquellas expresiones que suelen desestimarse como gestos o momentos políticos. Las propuestas de autores como Mouffe (1997) y Ranciere, Panagia y Bowlby (2001) ayudan a visibilizar el tipo de intercambios sociales que deberían tomarse en cuenta como base para definir la política dentro del enfoque y, en consecuencia, buscar mecanismos de articulación más adecuados a los contextos locales. Un esfuerzo que busque la articulación de varios actores, incluyendo a aquellos más vulnerables o marginados, debe tener en cuenta que dentro de la configuración social siempre habrá actores con roles específicos, marcados por una diferenciación en la forma en que acceden a los recursos y los derechos de que gozan.

Desde la mirada de la ecología política, la práctica de la gobernanza para la RRD se antoja especialmente compleja para regiones como la latinoame- 
ricana, en donde los riesgos y vulnerabilidades sociales frente a desastres hacen parte de los resultados esperados del proceso de producción y desarrollo. El reto está en crear las condiciones para llevar a cabo una mejor lectura de la agencia social. La gobernanza del riesgo inevitablemente debe construirse sobre espacios de confrontación del poder. En estas condiciones, habría que pensar en la conveniencia de inscribir los esfuerzos para la RRD como acompañamiento en las pugnas de comunidades y grupos diversos, frente a poderes hegemónicos que ahora operan como intermediarios en la relación sociedad-medioambiente. En este propósito, la adopción de perspectivas teórico-metodológicas de corte filosófico y social-comunitario - Borda o Habermas, por mencionar algunos - puede constituir un impulso importante al trabajo que sobre RRD se realiza dentro de las bases sociales.

\section{Referencias}

Aguirre, Benigno. 2004. "Los desastres en América Latina: vulnerabilidad y resistencia". Revista Mexicana de Sociología 66 (3): 485-510. https://bit.ly/3x45zBi.

Alimonda, Héctor. 2015. "Ecología política latinoamericana y pensamiento crítico: vanguardias arraigadas". Desenvolvimento e Meio Ambiente 35: 161-168. https://doi.org/10.5380/ dma.v35i0.44557.

Azamar, Aleida, y José Ignacio Ponce. 2015. "El neoextractivismo como modelo de crecimiento en América Latina". Economía y Desarrollo 154 (1): 185-198. https://bit.ly/2Tceh22.

Beck, Ulrich. 2006. La sociedad del riesgo: hacia la nueva modernidad. Barcelona: Paidós.

Blaikie, Piers. 1985. The Political Economy of Soil Erosion in Developing Countries. Nueva York: Longman.

Blaikie, Piers, Terry Cannon, Ian Davis y Ben Wisner. 1996. Vulnerabilidad y el entorno social, político y económico de los desastres. Bogotá: Tercer Mundo Editores.

Borda, Fals. 1992. "La ciencia y el pueblo. Nuevas reflexiones". En La investigación acción participativa: inicios y desarrollos, coordinado por María Cristina Salazar, 65-84. Bogotá: Editorial Popular.

Bravo, Elizabeth. 2018. Ecología política de los desastres. El terremoto del 2016 en Ecuador. Quito: Editorial Abya-Yala.

Bryant, Raymond, y Sinéad Bailey. 1997. Third World Political Ecology. Nueva York: Routledge. 


\section{Deysi Ofelmina Jerez-Ramírez y Rogelio Josué Ramos-Torres}

Cardona, Omar. 2001. "La necesidad de repensar de manera holística los conceptos de vulnerabilidad y riesgo". Ponencia presentada en el International Work-Conference in Disaster Theory and Practice, Wageningen. Accedido abril de 2021. https://n9.cl/jufz1.

Castro-Gómez, Santiago. 2007. "Michel Foucault y la colonialidad del poder". Tabula Rasa 6: 153-172. https://bit.ly/3cpEj8H.

Cohen, Charles, y Eric Werker. 2008. "The Political Economy of Natural Disasters”. Journal of Conflict Resolution 52 (6): 795-819. https://doi.org/10.1177/0022002708322157.

Delgado, Gian Carlo. 2013. “Por qué es importante la ecología política?”. Revista Nueva Sociedad 244: 47-60. https://bit.ly/3g2Ze3v.

Frenkel, Alejandro. 2019. "Disparen contra las olas: securitización y militarización de desastres naturales y ayuda humanitaria en América Latina". Íconos 64: 183-202. https://bit. ly/3g6XeHx.

Habermas, Jürgen. 2001. Teoría de la acción comunicativa I. Racionalización de la acción y racionalización social. Madrid: Taurus.

Jerez-Ramírez, Deysi. 2016. "Las nuevas dialécticas de la prevención y la participación comunitaria”. En Gestión social de desastres, cambio climático y políticas públicas en el siglo XXI. Contradicciones y perspectivas desde México, Indonesia, Estados Unidos y Cuba, coordinado por Daniel Rodríguez, 33-63. Ciudad de México: Universidad Nacional Autónoma de México.

Leff, Enrique. 2017. "Las relaciones de poder del conocimiento en el campo de la ecología política: una mirada desde el Sur". En Ecología política latinoamericana, pensamiento crítico, diferencia latinoamericana y rearticulación epistémica, coordinado por Héctor Alimonda, Catalina Toro y Facundo Martín, 129-165. Buenos Aires: CLACSO.

Luhmann, Niklas. 2006. Sociología del riesgo. Ciudad de México: Universidad Iberoamericana.

Macías, Jesús. 2015. "Crítica de la noción de resiliencia en el campo de estudios de desastres". Revista Geográfica Venezolana 56 (2): 309-325. https://bit.ly/3csksFG.

Mouffe, Chantal. 1999. El retorno de lo político. Buenos Aires: Paidós.

ONU Asamblea General. 1989. Decenio Internacional para la Reducción de los Desastres Naturales. 22 de diciembre. A/RES/44/236. Nueva York: ONU.

---. 1994. Estrategia y plan de acción de Yokohama para un mundo más seguro (Conferencia Mundial sobre la Reducción de los Desastres Naturales). Ginebra: ONU.

--- 2005. Marco de acción de Hyogo para 2005-2015: aumento de la resiliencia de las naciones y las comunidades ante los desastres (Conferencia Mundial sobre la Reducción de los Desastres). Kobe: ONU/EIRD. https://n9.cl/bujlb.

---. 2015. Marco de Sendai para la reducción del riesgo de desastres 2015-2030 (Tercera Conferencia Mundial sobre la Reducción de los Desastres). Ginebra: UNISDR. https:// n9.cl/q6kld.

ONU, y Oficina de las Naciones Unidas para la Reducción de Riesgos de Desastres (UNDRR). 2021. Informe de evaluación regional sobre el riesgo de desastres en América Latina y el Caribe. Panamá: UNDRR. https://n9.cl/oiih. 
Organización para la Cooperación y el Desarrollo Económicos (OCDE). 2014. Recomendación del consejo sobre la gobernanza de riesgos críticos. Accedido abril de 2021. https:// bit.ly/2 VgGrdy.

Ortega, Pere, y Tica Font. 2012. "Seguridad nacional, seguridad multidimensional, seguridad humana". Papeles de Relaciones Ecosociales y Cambio Global 119: 161-172. https://bit. $1 \mathrm{y} / 3 \mathrm{pE} 1 \mathrm{O} 3 \mathrm{~m}$.

Peeling, Mark, y Kathleen Dill. 2009. "Disaster Politics: Tipping Points for Change in the Adaptation of Sociopolitical Regimes". Progress in Human Geography 34 (1): 21-37. https://doi.org/10.1177/0309132509105004.

Pereira, Andrés, y Emmanuel Raju. 2020. "The Politics of Disaster Risk Governance and Neo-Extractivism in Latin America". Politics and Governance 8 (4): 220-231. https://doi. org/10.17645/pag.v8i4.3147.

Programa de las Naciones Unidas para el Desarrollo (PNUD). 1994. Informe sobre desarrollo humano. Nueva York: PNUD. https://n9.cl/154u0g.

---. 2019. Informe sobre desarrollo humano. Nueva York: PNUD. https://n9.cl/2k0a.

---.2021. Réduction des risques de catastrophe, gouvernance et integration. Nueva York: PNUD. https://n9.cl/nm87f.

Raffestin, Claude, y Octavio González. 2013. Por una geografía del poder. Michoacán: El Colegio de Michoacán.

Ranciere, Jacques, Davide Panagia y Rachel Bowlby. 2001. "Ten Theses on Politics". Theory and Event 5 (3): 14-26. https://doi.org/10.1353/tae.2001.0028.

Revet, Sandrine. 2011. "El mundo internacional de las catástrofes naturales". Política y Sociedad 48 (3): 537-54. https://doi.org/10.5209/rev_POSO.2011.v48.n3.36424.

Rodríguez, Daniel. 2017. Proyecto sobre protección civil y derechos humanos, Síntesis Ejecutiva. Ciudad de México. Comisión Nacional de los Derechos Humanos / Universidad Nacional Autónoma de México. https://n9.cl/ay60.

Rojas, Raúl. 1995. Guía para realizar investigaciones sociales. Ciudad de México: Plaza y Valdés Editores.

Romero Toledo, Hugo, y Hugo Romero Aravena. 2015. "Ecología política de los desastres: vulnerabilidad, exclusión socio-territorial y erupciones en la Patagonia chilena". Magallania 43 (3): 7-26. https://bit.ly/3xaKepU.

Sánchez, José. 2006. Gestión pública y governance. Toluca: Instituto de Administración Pública del Estado de México.

Sandoval, Vicente, y Juan Pablo Sarmiento. 2018. "Una mirada sobre la gobernanza del riesgo de desastres y la resiliencia urbana en América Latina y el Caribe: los asentamientos informales en la nueva agenda urbana". REDER 2 (1): 38-52. https://bit.ly/3v77X9k.

Sosa, Dulce María, y Verónica Vázquez. 2014. "Vulnerabilidad diferenciada por género, clase y edad. El impacto del huracán Karl en la Antigua, Veracruz". En Reflexiones y expresiones de la vulnerabilidad social en el Sureste de México, coordinado por Denisse Soares, 186-207. Morelos: Instituto Mexicano de Tecnología del Agua. 
Svampa, Maristella. 2019. Las fronteras del neoextractivismo en América Latina: conflictos socioambientales, giro ecoterritorial y nuevas dependencias. Guadalajara: CALAS.

Toro, Catalina. 2017. "Homenaje a Héctor Alimonda". En Ecología política Latinoamericana, pensamiento crítico, diferencia latinoamericana y rearticulación epistémica, coordinado por Héctor Alimonda, Catalina Toro y Facundo Martín, 21-28. Buenos Aires: CLACSO.

Wolf, Eric. 1990. "Facing Power, Old Insights New Questions". American Anthropologist, New Series 92 (3): 586-596. https://bit.ly/3w3fw1X. 